\title{
Matrix metalloproteinases 2 and 3 gene polymorphisms and the risk of target vessel revascularization after percutaneous coronary intervention: Is there still room for determining genetic variation of MMPs for assessment of an increased risk of restenosis?
}

\author{
J.J.W. Verschuren ${ }^{\mathrm{a}}$, M.L. Sampietro ${ }^{\mathrm{b}, \mathrm{j}}$, D. Pons ${ }^{\mathrm{a}, \mathrm{j}}$, S. Trompet ${ }^{\mathrm{a}}$, M.M. Ewing ${ }^{\mathrm{c}, \mathrm{k}}$, P.H.A. Quax ${ }^{\mathrm{c}, \mathrm{k}}$, \\ P. de Knijff ${ }^{b}$, A.H. Zwinderman ${ }^{\text {d }}$, R.J. de Winter ${ }^{\text {e,j }}$, R.A. Tio ${ }^{\text {f,j }}$, M.P. de Maat ${ }^{g}$, \\ P.A.F.M. Doevendans ${ }^{h, j}$ and J.W. Jukema ${ }^{a, i, j, k, *}$ \\ ${ }^{a}$ Department of Cardiology, Leiden University Medical Center, Leiden, The Netherlands \\ ${ }^{\mathrm{b}}$ Department of Human Genetics, Leiden University Medical Center, Leiden, The Netherlands \\ ${ }^{\mathrm{c}}$ Department of Surgery, Leiden University Medical Center, Leiden, The Netherlands \\ ${ }^{\mathrm{d}}$ Department of Medical Statistics, Academic Medical Center, Amsterdam, The Netherlands \\ ${ }^{\mathrm{e}}$ Department of Cardiology, Academic Medical Center, Amsterdam, The Netherlands \\ ${ }^{\mathrm{f}}$ Department of Cardiology, University Medical Center Groningen, Groningen, The Netherlands \\ ${ }^{\mathrm{g}}$ Department of Hematology, Erasmus Medical Center, Rotterdam, The Netherlands \\ ${ }^{\mathrm{h}}$ Department of Cardiology, University Medical Center Utrecht, Utrecht, The Netherlands \\ ${ }^{i}$ Durrer Center for Cardiogenetic Research, Amsterdam, The Netherlands \\ ${ }^{\mathrm{j}}$ The Interuniversity Cardiology Institute (ICIN), Utrecht, The Netherlands \\ ${ }^{\mathrm{k}}$ Einthoven Laboratory for Experimental Vascular Medicine, Leiden University Medical Center, Leiden, The \\ Netherlands
}

\begin{abstract}
Objective: Mixed results have been reported of matrix metalloproteinases (MMP) and their association with restenosis after percutaneous coronary intervention (PCI). The current study examines whether multiple single nucleotide polymorphisms (SNPs), covering the full genomic region of MMP2 and MMP3, were associated with restenosis in the GENDER study population. Methods and results: The GENetic DEterminants of Restenosis (GENDER) study enrolled 3104 consecutive patients after successful PCI. The primary endpoint was clinical restenosis, defined as target vessel revascularization (TVR), occurring in 9.8\% of the patients. From the Hapmap database, 19 polymorphisms of MMP2 and 11 of MMP3 were selected. Furthermore, in a subpopulation, a genome-wide association analysis (GWA) was performed. No significant association was found with any of the investigated SNPs, including the previously reported 5A/6A polymorphism (rs3025058), with regard to TVR using single SNP analysis or haplotype analysis.

Conclusion: We found no significant association of MMP2 or MMP3 with TVR with this SNP-broad gene approach. Although we did not test all the known polymorphisms of these genes, using tagging analyses we examined those SNPs covering all known
\end{abstract}

* Corresponding author: J.W. Jukema, MD, PhD, FESC, FACC, Professor of Cardiology, Chairman Leiden Vascular Medicine, Director Durrer Institute for Cardiogenetic Research Netherlands, Dept. of Cardiology, C5-P, Leiden University Medical Center, P.O. Box 9600, 2300 RC Leiden, The Netherlands. Tel.: +31 7152620 20; Fax: +31 7152668 85; E-mail: j.w.jukema@lumc.nl. 
haplotypes of MMP2 and MMP3 to conclude that these genes do not correlate with a genetic risk of coronary restenosis after successful PCI.

Keyword: Matrix metalloproteinases, percutaneous coronary intervention, restenosis, single nucleotide polymorphisms

\section{Introduction}

Matrix metalloproteinases (MMPs) form a family of over 20 zinc-dependent enzymes with proteolytic activity against a variety of extracellular matrix components including collagen, proteoglycans and elastin. All MMPs have distinct although overlapping substrate specificities [19]. Increased expression and activity of MMPs have been identified in various pathological processes, such as general inflammation, tumor metastasis, respiratory diseases, myocardial injury, vascular aneurysms, and vascular remodeling [29]. Vascular remodeling and inflammation are important features of restenosis after percutaneous coronary intervention (PCI) and several, mostly small, studies found associations of MMP plasma levels or genetic polymorphisms with restenosis $[5,9,12]$.

MMP2 (gelatinase A) is produced by vascular smooth muscle cells (VSMCs) to degrade basement membrane components and other matrix proteins during migration and proliferation of these cells. Different animal models for restenosis showed upregulation of MMP2 during intima formation [13,17]. Furthermore, MMP2 plasma levels were found to be increased after PCI and associated with restenosis $[7,9,16]$. Although genetic polymorphisms in the MMP2 gene have been associated with myocardial infarction (MI) $[8,26]$ and heart failure $[11,25]$, there are no studies published describing their association with coronary restenosis.

MMP3 (stromelysin-1) cleaves a wide variety of matrix proteins and is considered to reduce the matrix content of the vascular wall. Higher MMP3 expression is associated with the development of atherosclerotic plaques with less matrix protein and as a result smaller and more prone to rupture plaques (i.e. lipid rich plaques). On the other hand, low MMP3 expression genotypes are predisposed to develop relatively larger and more stable plaques (i.e. fibrotic plaques) [29]. However, in a MMP3 knockout mouse model, Johnson et al. found that in absence of MMP3 the plaques where not only larger but that they also had a lower smooth muscle cell content, making them less stable [14]. Part of their explanation was that MMP3 has a broad substrate specific, including activation of other MMPs and that therefore many mechanisms could account for the effects of MMP3 [14]. Functional studies have shown that the MMP3 -1612 5A/6A promoter polymorphism (rs3025058) is associated with decreased MMP3 expression levels $[3,18,28]$. In addition, several studies describe the association of this polymorphism with restenosis. For example, de Maat et al. found an association between the 6A6A MMP3 genotype and an increased risk of restenosis after balloon angioplasty [5]. The same conclusion was made by Humphries et al. [12]; however other studies could not identify this association $[4,10]$.

MMPs have been extensively studied on their association with different cardiovascular endpoints. The results with regard to restenosis have been mixed. To clarify these uncertainties we conducted this study in the large prospective GENetic DEterminants of Restenosis (GENDER) study population. With this study we systematically examined whether multiple single nucleotide polymorphisms (SNPs), covering the full genomic region of MMP2 and MMP3, were associated with clinical restenosis in the GENDER study population.

\section{Methods}

\subsection{Study design}

The present study population has been described previously [1]. In brief, the GENDER project was designed to study the association between various gene polymorphisms and clinical restenosis. All included patients were treated successfully for stable angina, non-ST-elevation acute coronary syndromes or silent ischemia by PCI in four out of the eight academic medical centers in the Netherlands. Patients treated for acute ST elevation myocardial infarction were excluded. Also excluded from analysis were patients suffering from events occurring within one month after the procedure, since these events were mostly attributable to sub-acute stent thrombosis or occluding dissections, and not to restenosis. During the study, no drug-eluting stents were used. Follow-up lasted for at least nine months, except when a coronary event occurred. Clinical restenosis, defined as renewed symptoms requiring 
Table 1

Demographic, clinical and lesion characteristics of the total GENDER population composed of 3,104 patients

\begin{tabular}{|c|c|c|c|}
\hline & Cases $(n=304)$ & Controls $(n=2,800)$ & $\mathrm{p}$-value \\
\hline Age (years) & $61.7 \pm 10.1$ & $62.2 \pm 10.8$ & 0.46 \\
\hline BMI $\left(\mathrm{kg} \cdot \mathrm{m}^{-2}\right)$ & $26.9 \pm 3.7$ & $27.0 \pm 3.9$ & 0.70 \\
\hline Male sex & $220(72.4 \%)$ & $1996(71.3 \%)$ & 0.69 \\
\hline Caucasian ancestry & $295(97.0 \%)$ & $2714(96.9 \%)$ & 0.92 \\
\hline Diabetes & $63(20.7 \%)$ & $390(13.9 \%)$ & 0.001 \\
\hline Hypercholesterolemia & $188(61.8 \%)$ & $1702(60.8 \%)$ & 0.72 \\
\hline Hypertension & $138(45.4 \%)$ & $1121(40.0 \%)$ & 0.07 \\
\hline Current smoker & $62(20.4 \%)$ & $700(25.0 \%)$ & 0.08 \\
\hline Family history of MI & $121(39.8 \%)$ & $977(34.9 \%)$ & 0.09 \\
\hline Previous MI & $109(35.9 \%)$ & $1130(40.4 \%)$ & 0.13 \\
\hline Previous PCI & $64(21.1 \%)$ & $493(17.6 \%)$ & 0.14 \\
\hline Previous CABG & $36(11.8 \%)$ & $340(12.1 \%)$ & 0.88 \\
\hline Stable angina & $198(65.1 \%)$ & $1881(67.2 \%)$ & 0.47 \\
\hline Acute coronary syndrome & $106(34.9 \%)$ & $974(31.2 \%)$ & 0.19 \\
\hline Multivessel disease & $148(48.7 \%)$ & $1284(45.9 \%)$ & 0.35 \\
\hline Peripheral vessel disease & $12(3.9 \%)$ & $92(3.3 \%)$ & 0.54 \\
\hline Lipid lowering medication & $171(56.3 \%)$ & $1516(54.1 \%)$ & 0.48 \\
\hline Restenotic lesions & $27(8.9 \%)$ & $181(6.5 \%)$ & 0.11 \\
\hline Total occlusions & $56(18.4 \%)$ & $372(13.3 \%)$ & 0.01 \\
\hline Type C lesion & $94(30.9 \%)$ & $708(25.3 \%)$ & 0.03 \\
\hline Proximal LAD & $70(23.0 \%)$ & $619(22.1 \%)$ & 0.71 \\
\hline $\mathrm{RCX}$ & $75(24.7 \%)$ & $764(27.3 \%)$ & 0.33 \\
\hline Stenting & $207(68.1 \%)$ & $2145(76.6 \%)$ & 0.001 \\
\hline Number of stents & $0.98 \pm 0.05$ & $1.01 \pm 0.01$ & 0.58 \\
\hline
\end{tabular}

Values were given as $\mathrm{n}(\%)$ or mean $\pm \mathrm{SD}$;

BMI, body mass index; MI, myocardial infarction; PCI, percutaneous coronary intervention; $\mathrm{CABG}$, coronary artery bypass graft; LAD, left anterior descending branch of the left coronary artery; RCX, circumflex branch of the left coronary artery.

p-value determined by Pearsons Chi-Square (discrete variables) or unpaired 2sided t-test (continuous variables).

target vessel revascularization (TVR), either by repeated PCI or CABG, was designated to be the primary endpoint. The study protocol met the criteria of the Declaration of Helsinki and was approved by the Medical Ethics Committees of each participating institution. Written informed consent was obtained from all participating patients in advance of the PCI procedure. Blood was collected in EDTA tubes at baseline and genomic DNA was extracted following standard procedures.

\subsection{Candidate gene approach}

SNPs of MMP2 and MMP3 were selected from the HapMap database (http://www.hapmap.org). Using the data from the CEU population, tagging SNPs, with a minor allele frequency $>5 \%$, were selected to cover each haplotype block within the genes (using Haploview program, version 4.1 [2]). Five SNPs in MMP2 were analyzed (rs243866, rs857403, rs243849, rs2287076 and rs10775332) and 2 SNPs in MMP3 (rs679620 and rs646910). Furthermore, the 5A6A promoter polymorphism of MMP3 (rs3025058) was added to the tagging SNPs. All SNPs were genotyped by matrix-assisted laser desorption/ionisation time-offlight (MALDI-TOF) mass spectrometry (MS), using the Sequenom MassARRAYtm methodology (Sequenom Inc, San Diego, CA, USA), following manufacturer's instructions. As quality controls, 368 (11\%) of the samples were genotyped in duplo. No inconsistencies were observed. All the blanks (5\%) were negative. Cluster plots were made of the signals from the low and the high mass allele. Independent researchers carried out scoring. Disagreements or vaguely positioned dots $(<1 \%)$ produced by Typer 4.0 (Sequenom Inc.) were left out of the results.

\subsection{Genome wide association study}

A genome wide association study was performed in a selection of cases and controls from the GENDER study and has been described previously [20]. In brief, cases and controls were selected from the total GENDER study population and matched by age, gender and other clinical factors such as diabetes and current smok- 
Table 2

Single nucleotide polymorphisms of MMP2 and the association with target vessel revascularization

\begin{tabular}{lccccccccc}
\hline SNP & Major allele & Minor allele & MAF & Approach & Rel. position & Gene region & HR & $95 \%$ CI & p-value \\
\hline rs893226 & G & T & 0.40 & GWAS & -10496 & & 1,00 & $(0.8401,20)$ & 0,97 \\
rs1005913 & A & C & 0.29 & GWAS & -8871 & Promoter & 0.99 & $(0.82-1.20)$ & 0.95 \\
rs1347653 & G & T & 0.17 & GWAS & -8352 & Promoter & 0.92 & $(0.72-1.17)$ & 0.42 \\
rs16955194 & G & A & 0.06 & GWAS & -8064 & Promoter & 1.38 & $(0.90-2.11)$ & 0.14 \\
rs243866 & G & A & 0.24 & Candid. & -1855 & Promoter & 0.90 & $(0.74-1.09)$ & 0.28 \\
rs857403 & A & T & 0.18 & Candid. & 3316 & Intron & 0.83 & $(0.67-1.04)$ & 0.11 \\
rs17301608 & C & T & 0.39 & GWAS & 5219 & Intron & 1.09 & $(0.92-1.29)$ & 0.32 \\
rs1053605 & C & T & 0.08 & GWAS & 6216 & Coding exon & 1.15 & $(0.84-1.57)$ & 0.40 \\
rs9302671 & G & T & 0.37 & GWAS & 8334 & Intron & 1.09 & $(0.92-1.30)$ & 0.32 \\
rs243849 & C & T & 0.17 & Candid. & 10313 & Coding exon & 0.82 & $(0.65-1.04)$ & 0.11 \\
rs243842 & T & C & 0.34 & GWAS & 14031 & Intron & 0.96 & $(0.801 .15)$ & 0.63 \\
rs183112 & G & A & 0.15 & GWAS & 14291 & Intron & 1.24 & $(0.97-1.58)$ & 0.09 \\
rs1992116 & C & T & 0.46 & GWAS & 14500 & Intron & 1.16 & $(0.98-1.37)$ & 0.09 \\
rs243840 & A & G & 0.20 & GWAS & 14768 & Intron & 0.83 & $(0.65-1.04)$ & 0.10 \\
rs2287076 & T & C & 0.47 & Candid. & 19066 & Intron & 1.17 & $(0.99-1.38)$ & 0.08 \\
rs243834 & A & G & 0.47 & GWAS & 23296 & Intron & 0.88 & $(0.74-1.04)$ & 0.14 \\
rs10775332 & C & T & 0.46 & Candid. & 23335 & Coding exon & 1.10 & $(0.93-1.30)$ & 0.22 \\
rs1861320 & G & T & 0.47 & GWAS & 27649 & Downstream & 1.12 & $(0.94-1.33)$ & 0.19 \\
rs8054459 & A & G & 0.44 & GWAS & 31158 & Downstream & 0.84 & $(0.71-1.01)$ & 0.06 \\
\hline
\end{tabular}

SNP, single nucleotide polymorphism; MAF, minor allele frequency; Rel., relative; HR, hazard ratio; CI, confidence interval; candid., candidate approach; GWAS, genome wide association scan.

Cox regression analysis with additive model with correction for sex, age and ethnicity in the GWAS SNPs and also correction for diabetes, total occlusion, stenting and type C lesions in the candidate SNPs.

Table 3

Single nucleotide polymorphisms of MMP3 and the association with target vessel revascularization

\begin{tabular}{|c|c|c|c|c|c|c|c|c|c|}
\hline SNP & Major allele & Minor allele & MAF & Approach & Rel. position & Gene region & HR & $95 \% \mathrm{CI}$ & p-value \\
\hline rs645419 & $\mathrm{A}$ & $\mathrm{G}$ & 0.48 & GWAS & -2045 & Promoter & 1.04 & $(0.87-1.25)$ & 0.66 \\
\hline rs3025058 & $5 \mathrm{~A}$ & $6 \mathrm{~A}$ & 0.50 & Candid. & -1676 & Promoter & 1.09 & $(0.92-1.28)$ & 0.33 \\
\hline rs522616 & $\mathrm{A}$ & $\mathrm{G}$ & 0.21 & GWAS & -772 & Promoter & 1.03 & $(0.83-1.27)$ & 0.80 \\
\hline rs679620 & A & $\mathrm{G}$ & 0.50 & Candid. & 657 & Coding exon & 1.07 & $(0.90-1.26)$ & 0.46 \\
\hline rs3025066 & A & $\mathrm{G}$ & 0.06 & GWAS & 3794 & Intron & 1.09 & $(0.78-1.54)$ & 0.61 \\
\hline rs566125 & $\mathrm{C}$ & $\mathrm{T}$ & 0.15 & GWAS & 3806 & Intron & 0.89 & $(0.69-1.15)$ & 0.38 \\
\hline rs646910 & $\mathrm{T}$ & A & 0.13 & Candid. & 4755 & Intron & 1.06 & $(0.83-1.35)$ & 0.67 \\
\hline rs595840 & $\mathrm{T}$ & $\mathrm{C}$ & 0.48 & GWAS & 9380 & Downstream & 1.03 & $(0.86-1.24)$ & 0.72 \\
\hline rs527832 & $\mathrm{C}$ & $\mathrm{T}$ & 0.12 & GWAS & 12393 & Downstream & 0.94 & $(0.72-1.23)$ & 0.67 \\
\hline rs1010698 & A & $\mathrm{C}$ & 0.48 & GWAS & 13256 & Downstream & 1.03 & $(0.86-1.24)$ & 0.72 \\
\hline rs1034375 & A & $\mathrm{C}$ & 0.07 & GWAS & 18805 & Downstream & 1.23 & $(0.90-1.67)$ & 0.19 \\
\hline
\end{tabular}

SNP, single nucleotide polymorphism; MAF, minor allele frequency; Rel., relative; HR, hazard ratio; CI, confidence interval; candid., candidate approach; GWAS, genome wide association scan.

Cox regression analysis with additive model with correction for sex, age and ethnicity in the GWAS SNPs and also correction for diabetes, total occlusion, stenting and type C lesions in the candidate SNPs.

ing that have been previously associated to restenosis. A genome-wide association analysis was conducted using the Illumina Human 610-Quad Beadchips following manufacturer's instructions. These beadchips contain 620,901 single nucleotide polymorphism (SNP) and copy number variants (CNV) probes, covering $89 \%$ of the common genetic variation in the European population at $\mathrm{r}^{2}>0.8$. After genotyping, samples and genetic markers were subjected to a stringent quality control protocol. Of the initially selected patients (321 cases and 620 controls), 295 cases and 571 controls fulfilled all criteria. The SNPs captured in the MMP2 region between positions 54060397 and 54102050 on chro- mosome 16 and the SNPs in the MMP3 region between positions 102206231 and 102225888 on chromosome 11 were selected for further analyses (see Tables 2 and 3 for rs numbers).

\subsection{Statistical analysis}

Of the baseline characteristics the discrete variables are expressed as counts or percentages and were compared with chi-square tests. Continuous variables are expressed as mean ( \pm standard deviation) and were compared by means with unpaired 2-sided t-test. Associations of the genotypes of the individual SNPs with 


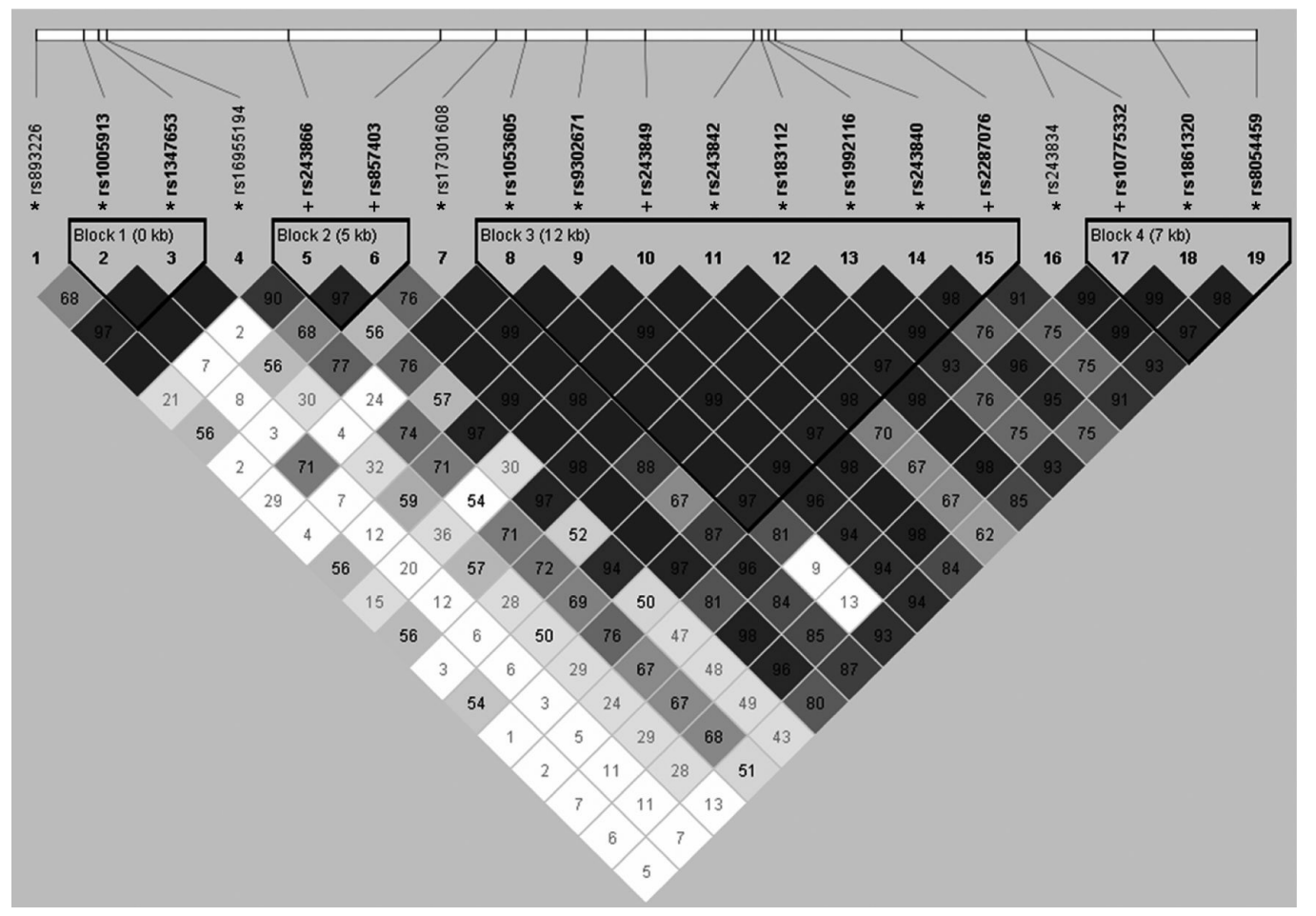

Fig. 1. Linkage disequilibrium structure of 19 MMP2 SNPs on chromosome 16 . Values shown in D', *SNP from GWAS, + SNP from candidate approach.

TVR were calculated assuming an additive model using a Cox proportional hazards model. We controlled for age, sex and ethnicity in the Cox-regression by including them as covariates. Furthermore, based on the baseline characteristics, we also corrected for diabetes, stenting, total occlusion and type $\mathrm{C}$ lesions in the analysis of the candidate approach SNPs. These additional corrections were not necessary for the GWAS SNPs, since that subpopulation was matched for known risk factors as described above. Recessive and dominant models were also tested. After Bonferroni correction for multiple testing, we considered a statistically significant association (at $p<0.05$ ) for any SNP with p-value $<0.0017$.

The program Haploview was used to estimate allele frequencies, test the consistency of the genotype frequencies at each SNP locus with Hardy-Weinberg equilibrium, and estimate and plot pairwise linkage disequilibrium (LD) measurements between SNPs.

Haplotypes and haplotype frequencies were calculated using PHASE software (http://www.stat.washing ton.edu/stephens/) [22-24]. Haplotypes with a frequency of less than $5 \%$ were combined and included in all analyses, without reporting the results. The haplotype analysis approach performed in this study as- sumes an additive effect of the haplotypes, and details of this approach have been described elsewhere [27]. Haplotype analyses were performed using STATA for haplotypes with a frequency $>5 \%$. Their effects were calculated using a Cox proportional hazards model including sex, age, and ethnicity and weighted for the probability of having the haplotype.

A p-value $<0.05$ was considered statistically significant. Statistical analysis was carried out using SPSS 17.0 for Windows.

\section{Results}

\subsection{Baseline characteristics}

A total of 3,146 patients had a complete follow-up (99.3\%) with a median duration of 9.6 months (interquartile range 3.9). Out of 3,146 patients 42 had an event in the first 30 days. These patients were excluded from further analysis, according to the protocol. Baseline characteristics of the population are shown in Table 1. Of the 3,104 patients, $304(9.8 \%)$ patients underwent TVR during follow-up. 51 patients died (1.6\%) and $22(0.7 \%)$ suffered from MI. In the total GENDER 


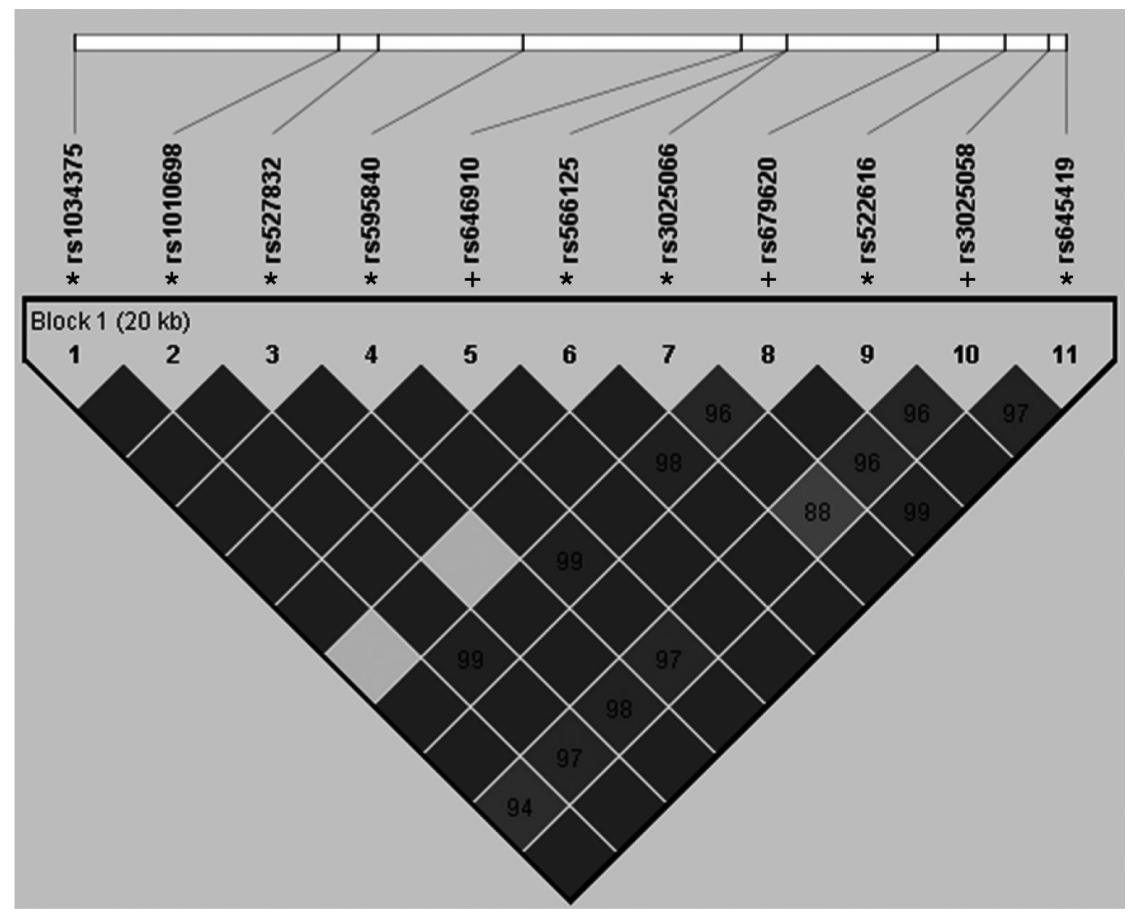

Fig. 2. Linkage disequilibrium structure of 11 MMP3 SNPs on chromosome 11. Values shown in D', * SNP from GWAS, + SNP from candidate approach.

population use of lipid lowering medication was equal in both cases and controls. Diabetes, total occlusion and C-type lesion were significantly more frequent in the cases ( $p$-value $=0.001,0.01$ and 0.03 respectively), whereas the percentage of patients receiving a coronary stent was significantly higher in the control group $(\mathrm{p}$-value $=0.001)$.

\subsection{Genotypic analyses}

The SNPs included in this study are listed in Tables 2 and 3 for MMP2 and MMP3, respectively. The 19 polymorphisms of MMP2 and 11 polymorphisms of MMP3 provided coverage of all haplotype blocks (Figs 1 and 2 , respectively) and were all in Hardy-Weinberg equilibrium ( $p$-value $>0.05$ ). Associations with TVR as a marker of restenosis were calculated assuming an additive model. No significant associations were observed between any of the MMP2 and MMP3 polymorphisms, including the previously reported $5 \mathrm{~A} / 6 \mathrm{~A}$ polymorphism (rs3025058), and TVR. Dominant and recessive models did not alter these effects (data not shown).

After analyzing the subpopulations of patients with ( $n=2352)$ or without $(n=752)$ receiving coronary stents during PCI, similar results were obtained. None of the SNPs was associated with TVR in neither of the subpopulations.

Linkage disequilibrium structure is shown in Fig. 1 and in Fig. 2. MMP2 included 4 haplotype blocks, whereas MMP3 had only 1. The haplotypes of the different blocks of MMP2 were calculated as shown in Table 4. The most frequent haplotype was used as reference. None of the haplotypes was significantly associated with the outcome parameter TVR. In line with the single SNP analysis, haplotype analysis of MMP3 also did not show any significant association with TVR (Table 5).

\section{Discussion}

Polymorphisms of MMPs have been associated with different cardiovascular events $[5,11,15,21,26$, 29]. Studies of their association with restenosis after PCI are however scarce. Most of these studies look into the relation of the 5A6A promoter polymorphism of MMP3 and restenosis, however those results are mixed $[4,5,10,12]$. The main finding of the present study is that after testing several polymorphisms in MMP2 and MMP3 using a candidate gene approach we did not find any significant association with coronary 
Table 4

Haplotype analysis of MMP2

\begin{tabular}{lccccc}
\hline & Haplotype & Frequency & HR & $95 \%$ CI & p-value \\
\hline Block 1 & AG* & $54.56 \%$ & & & \\
& CG & $28.12 \%$ & 1.00 & $(0.85-1.17)$ & 0.99 \\
& AT & $17.28 \%$ & 0.91 & $(0.75-1.11)$ & 0.37 \\
Block 2 & AA & $57.67 \%$ & & & \\
& GA $^{*}$ & $23.89 \%$ & 0.87 & $(0.72-1.05)$ & 0.15 \\
& AT & $18.05 \%$ & 0.82 & $(0.66-1.02)$ & 0.08 \\
Block 3 & CTCTATAC & $35.77 \%$ & & & \\
& CGCCACAT & $35.42 \%$ & 0.88 & $(0.73-1.06)$ & 0.18 \\
& CGTTGCGT & $15.43 \%$ & 0.90 & $(0.72-1.12)$ & 0.35 \\
& TGCTATAC & $7.11 \%$ & 1.08 & $(0.81-1.44)$ & 0.61 \\
Block 4 & TTA & $45.60 \%$ & & & \\
& CGG & $44.40 \%$ & 0.89 & $(0.76-1.05)$ & 0.16 \\
& CGA & $9.47 \%$ & 1.10 & $(0.86-1.42)$ & 0.44 \\
\hline
\end{tabular}

*wild type; HR, hazard ratio; CI, confidence interval.

Table 5

Haplotype analysis of MMP3

\begin{tabular}{lcccc}
\hline Haplotype & Frequency & HR & $95 \%$ CI & p-value \\
\hline AACTTCAAAAA & $42.43 \%$ & & & \\
ACTCTCAGGCG & $12.84 \%$ & 1.157 & $(0.84-1.59)$ & 0.37 \\
ACCCATAGACG & $11.99 \%$ & 0.994 & $(0.80-1.24)$ & 0.95 \\
ACCCTCAGGCG & $9.35 \%$ & 1.112 & $(0.87-1.42)$ & 0.40 \\
CCCCTCAGACG & $7.71 \%$ & 1.212 & $(0.93-1.58)$ & 0.16 \\
ACCCTCAGACG & $5.72 \%$ & 1.059 & $(0.78-1.44)$ & 0.72 \\
AACTTCGAAAA & $5.59 \%$ & 1.105 & $(0.87-1.41)$ & 0.42 \\
\hline
\end{tabular}

* wild type; HR, hazard ratio; CI, confidence interval.

restenosis. Since in the meantime a GWAS was carried out in a subpopulation of the GENDER study [20], we decided to include the genotyped polymorphisms in the genomic regions of both MMP2 and MMP3 in our analyses. None of the SNPs was associated with restenosis. Combining both approaches strengthened our findings of the lack of association between the two MMP genes and coronary restenosis after successful PCI.

Previously, Hojo et al. reported a significant association of increased serum level of MMP2 four hours after PCI and the occurrence of restenosis [9]. Their study population was however very small and the patient characteristics of the 29 patients with follow-up were not well described. Recently, Katsaros et al. reported that in a population of 85 patients with stable angina pectoris receiving DES, increased MMP2 serum activity was associated with dramatically increased restenosis rates [16]. Although these results look promising for possible identification of patients prone to develop restenosis, larger studies are needed to replicate these results. In our, much larger patient population, we could not confirm their results with genotypic data.
The increased risk of restenosis with the 6A6A MMP3 genotype was described by de Maat et al. and Humphries et al. in patients after balloon angioplasty without stenting $[5,12]$. The latter study also analyzed a subpopulation of patients who received an intracoronary stent and they could not identify an association with the 6A6A MMP3 genotype and restenosis. This difference probably indicates that different processes are involved in these two patient groups. However, since balloon angioplasty without stenting becomes more rare, the clinical relevance of this finding is decreasing. In the GENDER no significant association with TVR of any of the SNPs was detected in neither of the subpopulations. Moreover, the 5A6A polymorphism was also not related to restenosis, not in the total population nor in one of the subgroups.

In the GENDER study all patients that were stented received one or more bare-metal stents (BMS). It could be argued that a possible role of investigated MMPs might be ascribed in restenosis in a patient group receiving drug-eluting stents (DES). This is however highly unlikely since DES are associated with a reduction in the development of restenosis, which would more likely diminish a possible genotype-dependent difference 
in neointima formation. Definite data on this topic is however lacking.

A limitation of our study is that the patients included in the GENDER study were mostly patients with a Caucasian ancestry (96.9\%). Different results may be obtained in other ethnic groups. Furthermore, we did not test all known polymorphisms in the two genes of interest, but since SNPs were selected taking into account the LD measurements, all haplotype blocks were covered. Moreover, no significant associations were obtained by means of haplotype analysis. We therefore think it is valid to conclude that no major effects are to be expected from these genes. Also, our findings are based on one single study. However, since the GENDER study is a large prospective follow-up study and taken in account that the previous reports are heterogeneous and in smaller patients groups, the power of these results is large enough to draw these conclusions.

In conclusion, with this study we provide evidence that, with regard to restenosis, a solid association with genetic variation of MMP2 and MMP3 is absent. Although we did not test all the known polymorphisms of the MMP2 and MMP3 genes, using tagging analyses we examined those SNPs covering all known haplotypes of MMP2 and MMP3 to conclude that genetic variation of these genes does not correlate with a genetic risk of coronary restenosis after successful PCI. As the search for genetic factors involved in the process of restenosis continues, we merely exclude genetic variation in two previously proposed candidate genes for involvement in the increased risk for restenosis, so future research may focus on other targets.

\section{References}

[1] W.R. Agema, P.S. Monraats, A.H. Zwinderman, R.J. de Winter, R.A. Tio, P.A. Doevendans, J. Waltenberger, M.P. de Maat, R.R. Frants, D.E. Atsma, A. van der Laarse, E.E. van der Wall and J.W. Jukema, Current PTCA practice and clinical outcomes in The Netherlands: the real world in the pre-drugeluting stent era, Eur Heart J 25 (2004), 1163-1170.

[2] J.C. Barrett, B. Fry, J. Maller and M.J. Daly, Haploview: analysis and visualization of LD and haplotype maps, Bioinformatics 21 (2005), 263-265.

[3] S. Beyzade, S. Zhang, Y.K. Wong, I.N. Day, P. Eriksson and $\mathrm{S}$. Ye, Influences of matrix metalloproteinase-3 gene variation on extent of coronary atherosclerosis and risk of myocardial infarction, J Am Coll Cardiol 41 (2003), 2130-2137.

[4] K.R. Chiou, S.L. Chung and M.J. Charng, 5A/6A polymorphism of the stromelysin-1 gene and angiographic restenosis after coronary artery stenting, J Chin Med Assoc 68 (2005), $506-512$.
[5] M.P. de Maat, J.W. Jukema, S. Ye, A.H. Zwinderman, P.H. Moghaddam, M. Beekman, J.J. Kastelein, A.J. van Boven, A.V. Bruschke, S.E. Humphries, C. Kluft and A.M. Henney, Effect of the stromelysin-1 promoter on efficacy of pravastatin in coronary atherosclerosis and restenosis, Am J Cardiol $\mathbf{8 3}$ (1999), 852-856.

[6] M. Fatar, M. Stroick, M. Steffens, E. Senn, B. Reuter, S. Bukow, M. Griebe, A. Alonso, P. Lichtner, P. Bugert, T. Meitinger, T.F. Wienker and M.G. Hennerici, Singlenucleotide polymorphisms of MMP-2 gene in stroke subtypes, Cerebrovasc Dis 26 (2008), 113-119.

[7] J. Ge, C. Shen, C. Liang, L. Chen, J. Qian and H. Chen, Elevated matrix metalloproteinase expression after stent implantation is associated with restenosis, International Journal of Cardiology 112 (2006), 85-90.

[8] M.A. Hlatky, E. Ashley, T. Quertermous, D.B. Boothroyd, P. Ridker, A. Southwick, R.M. Myers, C. Iribarren, S.P. Fortmann and A.S. Go, Matrix metalloproteinase circulating levels, genetic polymorphisms, and susceptibility to acute myocardial infarction among patients with coronary artery disease, Am Heart J 154 (2007), 1043-1051.

[9] Y. Hojo, U. Ikeda, T. Katsuki, O. Mizuno, H. Fujikawa and K. Shimada, Matrix metalloproteinase expression in the coronary circulation induced by coronary angioplasty, Atherosclerosis 161 (2002), 185-192.

[10] P. Hoppmann, W. Koch, A. Schomig and A. Kastrati, The $5 \mathrm{~A} / 6 \mathrm{~A}$ polymorphism of the stromelysin-1 gene and restenosis after percutaneous coronary interventions, Eur Heart $J \mathbf{2 5}$ (2004), 335-341.

[11] Y. Hua, L. Song, N. Wu, X. Lu, X. Meng, D. Gu and Y. Yang, Polymorphisms of MMP-2 gene are associated with systolic heart failure risk in Han Chinese, Am J Med Sci 337 (2009), 344-348.

[12] S. Humphries, C. Bauters, A. Meirhaeghe, L. Luong, M. Bertrand and P. Amouyel, The 5A6A polymorphism in the promoter of the stromelysin-1 (MMP3) gene as a risk factor for restenosis, Eur Heart J 23 (2002), 721-725.

[13] C. Johnson and Z.S. Galis, Matrix metalloproteinase-2 and -9 differentially regulate smooth muscle cell migration and cellmediated collagen organization, Arterioscler Thromb Vasc Biol 24 (2004), 54-60.

[14] J.L. Johnson, S.J. George, A.C. Newby and C.L. Jackson, Divergent effects of matrix metalloproteinases 3, 7, 9, and 12 on atherosclerotic plaque stability in mouse brachiocephalic arteries, Proc Natl Acad Sci U S A 102 (2005), 15575-15580.

[15] R.C. Kaplan, N.L. Smith, S. Zucker, S.R. Heckbert, K. Rice and B.M. Psaty, Matrix metalloproteinase-3 (MMP3) and MMP9 genes and risk of myocardial infarction, ischemic stroke, and hemorrhagic stroke, Atherosclerosis 201 (2008), 130-137.

[16] K.M. Katsaros, S.P. Kastl, G. Zorn, G. Maurer, J. Wojta, K. Huber, G. Christ and W.S. Speidl, Increased Restenosis Rate After Implantation of Drug-Eluting Stents in Patients With Elevated Serum Activity of Matrix Metalloproteinase-2 and -9, JACC: Cardiovascular Interventions 3 (2010), 90-97.

[17] M. Kuzuya, S. Kanda, T. Sasaki, N. Tamaya-Mori, X.W. Cheng, T. Itoh, S. Itohara and A. Iguchi, Deficiency of gelatinase a suppresses smooth muscle cell invasion and development of experimental intimal hyperplasia, Circulation 108 (2003), 1375-1381.

[18] T.L. Medley, B.A. Kingwell, C.D. Gatzka, P. Pillay and T.J. Cole, Matrix metalloproteinase- 3 genotype contributes to agerelated aortic stiffening through modulation of gene and protein expression, Circ Res 92 (2003), 1254-1261. 
[19] A.C. Newby, Matrix metalloproteinases regulate migration, proliferation, and death of vascular smooth muscle cells by degrading matrix and non-matrix substrates, Cardiovasc Res 69 (2006), 614-624.

[20] M.L. Sampietro, D. Pons, K.P. de, P.E. Slagboom, A. Zwinderman and J.W. Jukema, A genome wide association analysis in the GENDER study, Neth Heart J 17 (2009), 262-264.

[21] A. Schwarz, W. Haberbosch, H. Tillmanns and A. Gardemann, The stromelysin-1 5A/6A promoter polymorphism is a disease marker for the extent of coronary heart disease, Dis Markers 18 (2002), 121-128.

[22] M. Stephens, N.J. Smith and P. Donnelly, A new statistical method for haplotype reconstruction from population data, $\mathrm{Am}$ J Hum Genet 68 (2001), 978-989.

[23] M. Stephens and P. Donnelly, A comparison of bayesian methods for haplotype reconstruction from population genotype data, Am J Hum Genet 73 (2003), 1162-1169.

[24] M. Stephens and P. Scheet, Accounting for decay of linkage disequilibrium in haplotype inference and missing-data imputation, Am J Hum Genet 76 (2005), 449-462.

[25] A. Vasku, M. Goldbergova, L.I. Holla, L. Spinarova, J. Spinar,
J. Vitovec, and J. Vacha, Two MMP-2 promoter polymorphisms $(-790 \mathrm{~T} / \mathrm{G}$ and $-735 \mathrm{C} / \mathrm{T})$ in chronic heart failure, Clin Chem Lab Med 41 (2003), 1299-1303.

[26] A. Vasku, M. Goldbergova, H.L. Izakovicova, L. Siskova, L. Groch, M. Beranek, S. Tschoplova, V. Znojil and J. Vacha, A haplotype constituted of four MMP-2 promoter polymorphisms $(-1575 \mathrm{G} / \mathrm{A},-1306 \mathrm{C} / \mathrm{T},-790 \mathrm{~T} / \mathrm{G}$ and $-735 \mathrm{C} / \mathrm{T})$ is associated with coronary triple-vessel disease, Matrix Biol 22 (2004), 585-591.

[27] S. Wallenstein, S.E. Hodge and A. Weston, Logistic regression model for analyzing extended haplotype data, Genet Epidemiol 15 (1998), 173-181.

[28] S. Ye, P. Eriksson, A. Hamsten, M. Kurkinen, S.E. Humphries and A.M. Henney, Progression of coronary atherosclerosis is associated with a common genetic variant of the human stromelysin-1 promoter which results in reduced gene expression, J Biol Chem 271 (1996), 13055-13060.

[29] S. Ye, Influence of matrix metalloproteinase genotype on cardiovascular disease susceptibility and outcome, Cardiovasc Res 69 (2006), 636-645. 


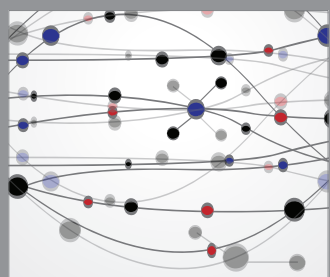

The Scientific World Journal
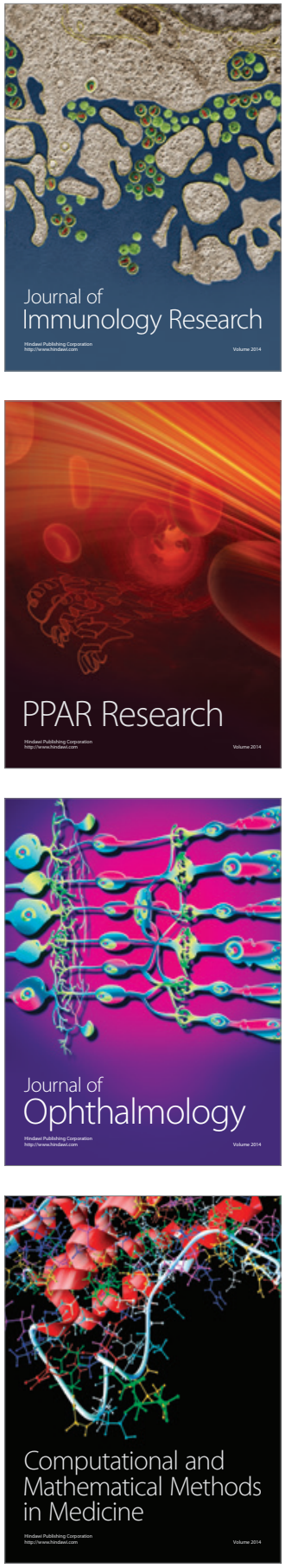

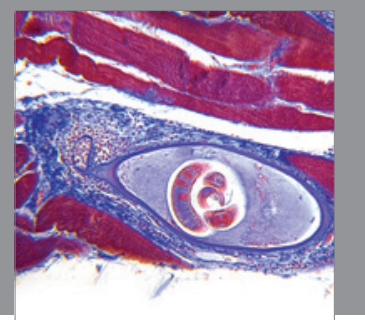

Gastroenterology

Research and Practice
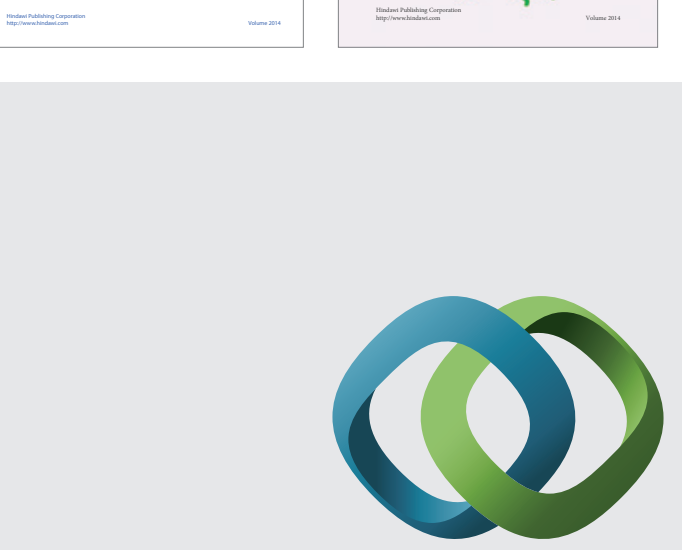

\section{Hindawi}

Submit your manuscripts at

http://www.hindawi.com
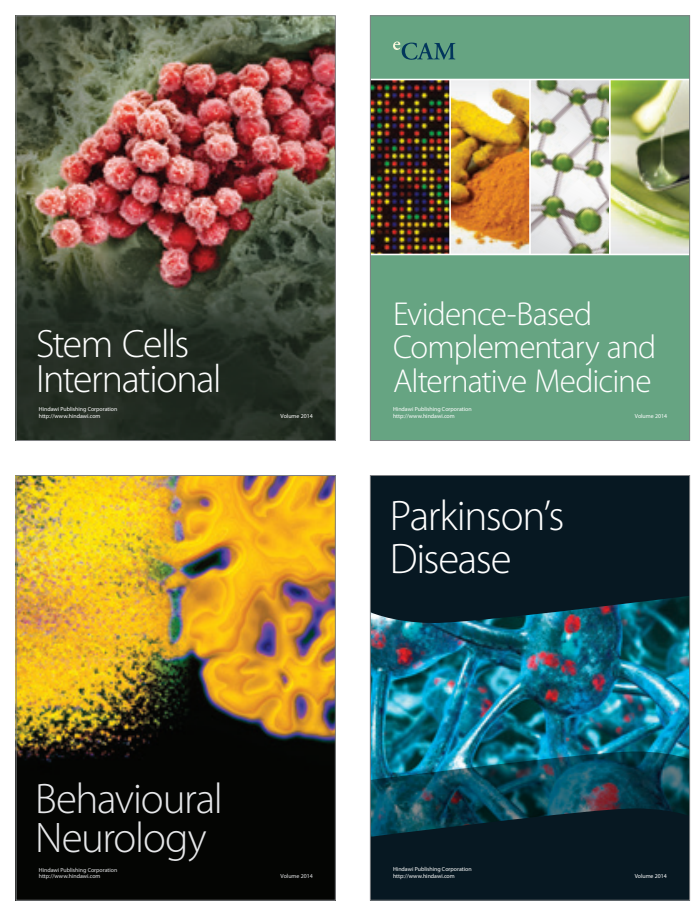

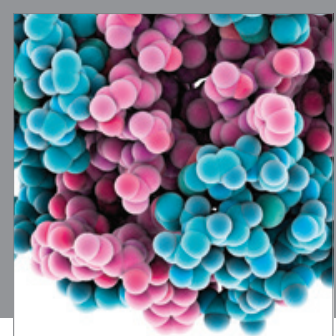

Journal of
Diabetes Research

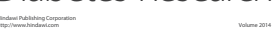

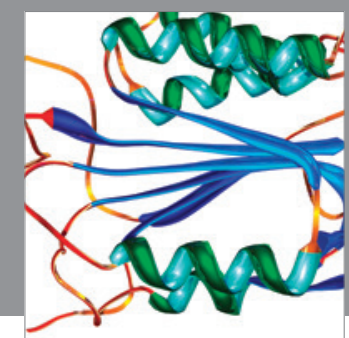

Disease Markers
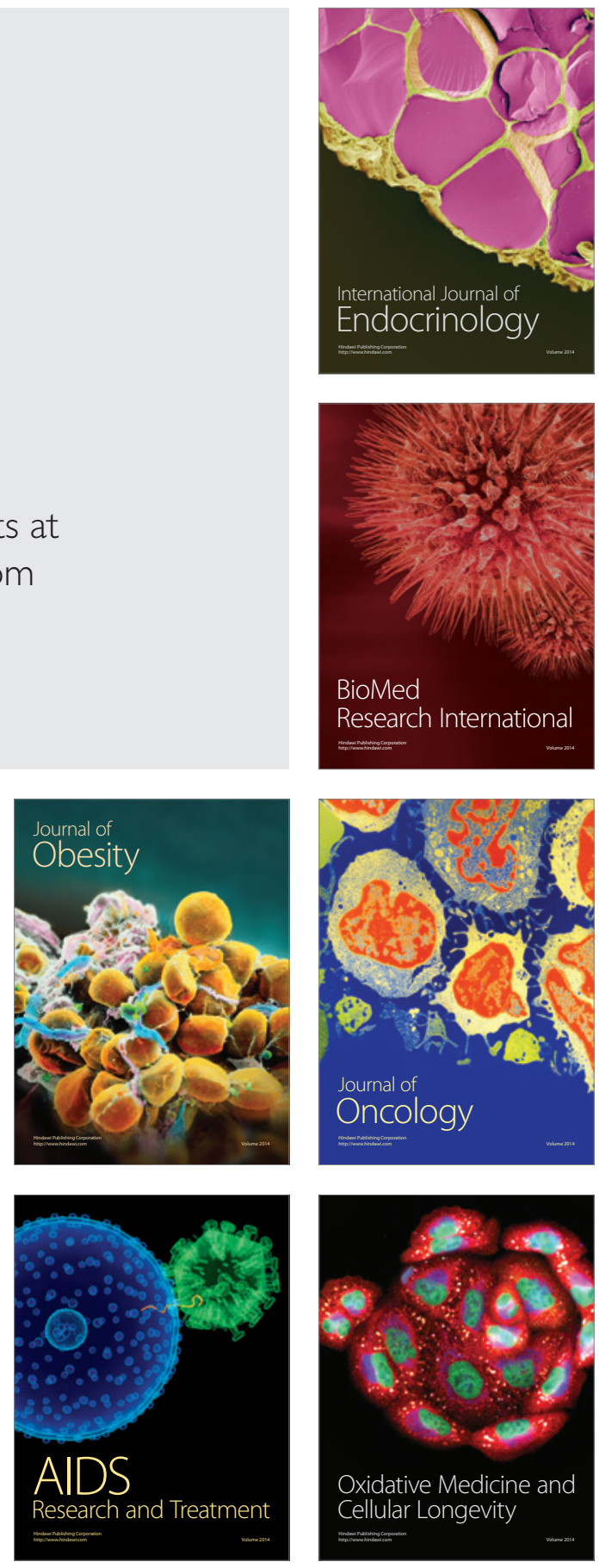